\title{
A Frequency-Domain Data-driven Adaptive Iterative Learning Control Approach: With Application to Wafer Stage
}

\author{
Xuewei Fu, Xiaofeng Yang, Pericle Zanchetta, Fellow, Yang Liu, Member, Chenyang Ding, \\ Mi Tang, Member, and Zhenyu Chen
}

\begin{abstract}
The feedforward control is becoming increasingly important in ultra-precision stages. However, the conventional model-based methods can not achieve expected performance in new-generation stages since it is hard to obtain the accurate plant model due to the complicated stage dynamical properties. To tackle this problem, this paper develops a model-free data-driven adaptive iterative learning approach that is designed in the frequency-domain. Explicitly, the proposed method utilizes the frequency-response data to learn and update the output of the feedforward controller online, which benefits that both the structure and parameters of the plant model are not required. An unbiased estimation method for the frequency response of the closed-loop system is proposed and proved through the theoretical analysis. Comparative experiments on a linear motor confirm the effectiveness and superiority of the proposed method, and show that it has the ability to avoid the performance deterioration caused by the model mismatch with the increasing iterative trials.
\end{abstract}

Index Terms-adaptive ILC, data-driven, frequency domain, feedforward control, linear motor.

\section{INTRODUCTION}

$\mathbf{N}$ OWADAYS, the ultra-precision motion stages have been widely applied in many nanoscale manufacturing industries like IC manufacturing [1]. The precision motion stage has

Manuscript received April 29, 2020; revised July 14, 2020 and August 17, 2020; accepted August 24, 2020. This work was supported in part by the National Science and Technology Major Project of China 2017ZX02101007, in part by the research project of Science and Technology Commission of Shanghai Municipality 18DZ1112600, and in part by the Pioneering Project of Academy for Engineering and Technology, Fudan University JIH2310101/011. (Corresponding author: Xiaofeng Yang)

X. Fu and X. Yang are with the State Key Laboratory of ASIC \& System, School of Microelectronics, Fudan University, Shanghai, 200433, China. (email: catfxw@163.com; xf_yang@fudan.edu.cn)

P. Zanchetta is with the Department of Electrical and Electronic Engineering, University of Nottingham, Nottingham, NG7 2RD, UK, and the Department of Electrical, Computer and Biomedical Engineering, University of Pavia, Pavia, 27100, Italy. (e-mail: pericle.zanchetta@nottingham.ac.uk)

Y. Liu and Z. Chen are with the Center of Ultra-Precision Optoelectronic Instrument Engineering, Harbin Institute of Technology, Harbin, 150080, China. (e-mail: hitlg@hit.edu.cn; hitczy@163.com)

C. Ding is with the Academy for Engineering \& Technology, Fudan University, Shanghai, 200433, China. (e-mail: dingcy@ fudan.edu.cn)

M. Tang is with the Power Electronics, Machine and Control Group, University of Nottingham, Nottingham, NG7 2RD, UK. (e-mail: mi.tang2@nottingham.ac.uk) a direct influence on the product quality [2], [3], which leads to higher performance requirements for the stages, such as higher tracking accuracy, larger velocity and acceleration. In the control system of the precision motion stages, the feedback control is usually used to suppress the unstructured external disturbance and the system uncertainty, while the feedforward control is used to compensate for the orderly disturbance, such as thrust ripple [4]. Usually, the control bandwidth is expected to be as high as possible, but it is limited by the mechanical resonance, the time delay of the drive system, the measurement bandwidth and so on. As a result, the feedforward control is necessary for high-performance motion stages as it can lower the requirements for the feedback control loop [5]. In a word, improvement in feedforward control is a significant step toward meeting higher performance in the next generation industrial precision motion stages [6]-[8].

As a popular feedforward method, iterative learning control (ILC) approach is widely applied in various scenarios [9]. The ILC could improve the tracking performance of the servo system by learning from the data gathered over the past trials [10], which is particularly effective in practical cases with the repetitive trajectory and the external disturbance. The ILC methods can be summarized into model-free ILC and modelbased ILC according to whether the plant model is required. Model-based ILC methods make use of model information so that it could achieve good tracking performance and high convergence speed. The most well-known model-based ILC methods applied to precision motion control such as the wafer stage control are mainly represented by the inversion-based ILC methods [11]-[13], Q-ILC [14], projection-based ILC [15], and so on. But the model used in the design process needs to be chosen properly as it plays an important role in the convergence properties. However, there exists flexible dynamical behavior like the small damping or the low resonant frequency in the next-generation precision motion stages [16], so the model-based ILC could hardly achieve the expected performance. In contrast to model-based ILC, model-free ILC methods seem to be more applicable for the future flexible precision motion stages since they do not require a plant model. However, the system information is not fully utilized. So the conventional model-free ILC [17]-[19] needs more iterative trials to tune the parameters for good performance and could hardly achieve the same performance as those modelbased methods. 
Therefore, a tradeoff has to be made between requiring no plant model and fully utilizing the system information to achieve higher performance of both tracking and convergence. To tackle this problem, the frequency-domain based ILC (FDILC) attracts more extensive research interest as it can make full use of the frequency-response information of the system, and no parameterized plant model is required. There are several achievements in FD-ILC. In [20], an FD-ILC method was proposed for the AFM piezoscanner and experimental results showed that the proposed method can significantly reduce the dynamic coupling errors. However, a small learning gain was chosen in [20] to keep the stability in the presence of the noise, which results in slower convergence speed. In [21], an inversion-based FD-ILC updating the inverse model by using the input-output data was presented for an AFM system and experimental results confirmed its ability to improve the tracking performance and convergence speed. Although there existed an updating for the learning law in [21], this proposed algorithm could not achieve an unbiased estimation of the inverse model and its estimation would certainly be affected by the noise. Additionally, there are some ILC methods designed in the frequency domain [22]-[24], but their learning laws were calculated based on the nominal plant model which would probably result in a limitation on tracking performance due to the model error.

Aforementioned analyses motivate the paper to propose a model-free frequency-domain adaptive ILC method (FDAILC). The contribution of this paper is threefold. First, a theoretical framework is developed for frequency-domain ILC with adaptive updating method. During the iterative process, the proposed FD-AILC method not only updates the ILC output, but also updates the ILC learning law. Furthermore, the criteria for accelerating convergence are proposed, which enables the possibility of achieving higher performance and increasing the convergence speed simultaneously. Second, an unbiased estimation method to solve the influence of measurement noise is proposed. Finally, an application to a linear motor of the wafer stage is presented to compare the proposed method with the model-based ILC methods, which demonstrates the effectiveness and superiority of the proposed method.

The rest of the paper is organized as follows. The problem statement is formulated in Section II. The frequency-domain data-driven adaptive ILC method and convergence analysis are presented in Section III. Simulation and Experimental results are presented with discussions in Section IV. The conclusions are drawn in Section V.

\section{Problem Statement}

The schematic diagram of the ILC approach widely applied to precision motion stage is shown in Fig. 1, where $r$ denotes the system reference trajectory, $y_{r}$ denotes the real system trajectory, $d$ denotes external disturbance, and $n$ denotes the measurement noise. $C_{L}$ is the iterative learning controller, $C_{f b}$ is the feedback controller, and $P$ is the plant model. It is noted that the system reference trajectory $r$ is set to be repetitive through the whole iterative process so there is no superscript

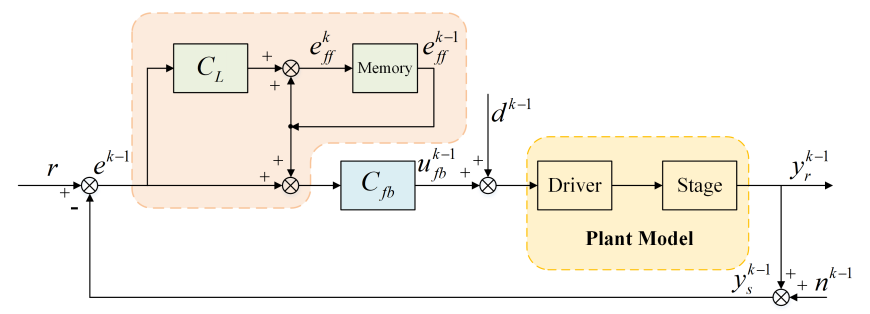

Fig. 1: Schematic diagram of the control system using the ILC method.

for $r$. From the basic principle of ILC, it can be obtained as follows.

$$
e_{f f}^{k}=e_{f f}^{k-1}+C_{L} \cdot e^{k-1}
$$

where, $e_{f f}^{k}$ is the ILC controller's output after the $k$-th iteration, and $e^{k}$ is the system tracking error after the $k$-th iteration.

Based on the former analysis, the conclusion can be drawn that the inversion-based ILC has the ability to improve the convergence speed. Therefore, this paper will focus on analyzing the ILC approach based on the inversion-model and further improving it by mapping the designing method from the time domain to the frequency domain. When $C_{L}=$ $\left(\left(C_{f b} \cdot P\right) /\left(1+C_{f b} \cdot P\right)\right)^{-1}$, the system can realize one-step convergence theoretically. However, in practice, there exists uncertainty in the controlled object and noise in the system. Filters are inevitably involved in the system, which causes that the system is unable to realize one-step convergence and the tracking accuracy is limited.

Therefore, it is considered to map the inversion-based ILC method to the frequency domain and design the iterative learning controller based on frequency-response data, namely as frequency-domain ILC (FD-ILC). It is defined as

$$
\begin{aligned}
C_{L}^{k}(\omega) & =\rho(\omega) \cdot \hat{T}_{k-1}^{-1}(\omega) \\
\hat{T}(\omega) & =\left(\frac{C_{f b}(\omega) \cdot P(\omega)}{1+C_{f b}(\omega) \cdot P(\omega)}\right)_{e s t}
\end{aligned}
$$

where, $\hat{T}(\omega)$ is the estimation of the closed-loop system frequency response which is obtained from the frequencyresponse test on the actual system, and $\rho(\omega)$ is the positive real regulator at the frequency point $\omega$, whose range is $\rho(\omega) \in(0,1]$. Therefore,

$$
e_{f f}^{k}(\omega)=e_{f f}^{k-1}(\omega)+C_{L}^{k}(\omega) \cdot e^{k-1}(\omega)
$$

where $\omega$ is selected according to the frequency points obtained from FFT calculation to the reference trajectory $r . e^{k-1}(\omega)$ is FFT of the time-domain error $e^{k-1}(t)$. The convergence condition of the FD-ILC method can be referred to [20] in detail.

Compared with inversion-based ILC method based on transfer function (TF-ILC), the FD-ILC method has the advantages that no transfer function model is required, no additional system time-delay compensation is needed, and the ideal filter can be realized by using frequency truncation. The FD-ILC 
method can effectively avoid the problem that the accurate plant model is difficult to obtain.

However, using the iterative learning law shown in (2) and (3), the obtained FD-ILC output is not completely accurate, which is due to the following reasons. Firstly, there exists external disturbance and measurement noise when testing the system frequency response. Secondly, it is hard to describe the system's high-order uncertainty. And thirdly, the frequency points obtained in the frequency-response test and the frequency points of the reference trajectory cannot be aligned precisely. So it needs to estimate the compensation value at the points of the required reference frequency through interpolation. That leads to a mismatch between the real value and the estimation value, which would introduce error into the FD-ILC output.

Therefore, given the drawbacks of FD-ILC method, a frequency-domain data-driven adaptive ILC method (FDAILC) is proposed, which can effectively make up for shortcomings of FD-ILC by updating the learning law using the data-driven adaptive method.

\section{Frequency-DOMAIN DATA-DRIVEn AdAPTIVE ILC}

The error of the closed-loop system frequency response is assumed to be multiplicative, and it is defined as

$$
\Delta T_{k}(\omega)=\frac{T(\omega)}{\hat{T}_{k}(\omega)}
$$

where, $T=C_{f b} P /\left(1+C_{f b} P\right)$.

According to the Fig. 1, there is

$$
\begin{aligned}
e^{k} & =r-\left(y_{r}^{k}+n^{k}\right) \\
& =r-\left[\left(e^{k}+e_{f f}^{k}\right) C_{f b} P+P \cdot d^{k}+n^{k}\right]
\end{aligned}
$$

Then $e^{k}$ can be deduced as

$$
e^{k}=S \cdot r-T \cdot e_{f f}^{k}-G_{P} \cdot d^{k}-S \cdot n^{k}
$$

where, $S=1 /\left(1+C_{f b} P\right)$ is the system sensitivity function and $G_{P}$ is defined as $G_{P}=P /\left(1+C_{f b} P\right)$.

(2) and (3) are substituted into (6) and the result below can be obtained.

$$
\begin{aligned}
e^{k}= & S \cdot r-T \cdot e_{f f}^{k-1}-\rho \cdot \Delta T_{k-1} \cdot e^{k-1} \\
& -G_{P} \cdot d^{k}-S \cdot n^{k}
\end{aligned}
$$

Meanwhile according to (6), $e^{k-1}$ can be written into

$$
e^{k-1}=S \cdot r-T \cdot e_{f f}^{k-1}-G_{P} \cdot d^{k-1}-S \cdot n^{k-1}
$$

The external disturbance $d^{k}$ can be rewritten into $d^{k}=$ $d_{r}+d_{n}^{k}$, where $d_{r}$ denotes the repetitive disturbance and $d_{n}^{k}$ denotes the nonrepetitive disturbance of the $k$-th iterative trial. Combining (7) with (8), there is

$$
\begin{gathered}
e^{k}(\omega)=\left[1-\rho(\omega) \cdot \Delta T_{k-1}(\omega)\right] \cdot e^{k-1}(\omega) \\
-S(\omega) \cdot\left[\tilde{n}^{k}(\omega)-\tilde{n}^{k-1}(\omega)\right]
\end{gathered}
$$

where the expression of $\tilde{n}^{k}(\omega)$ is

$$
\tilde{n}^{k}(\omega)=n^{k}(\omega)+P(\omega) \cdot d_{n}^{k}(\omega)
$$

When $\rho(\omega)=1$ and there is no system model error, that is $\Delta T(\omega)=1$, the system can realize one-step convergence. But there must be errors of the frequency response and $\Delta T(\omega)$ cannot be equal to 1 . If $|\Delta T(\omega)|$ approaches 1 , then $\rho(\omega)$ can be selected to be as large as possible, and the convergence rate of the algorithm will be faster. Therefore, $|\Delta T(\omega)|$ need to be improved as much as possible to approach 1 so that it can improve the convergence rate.

Based on the above analysis, the FD-ILC method is improved to update $\hat{T}(\omega)$ in addition to the output of the ILC controllers during each iteration. In this way, more accurate frequency-response data can be obtained in the iterative process, which can improve the control performance effectively. And the iterative learning law is modified as

$$
\left\{\begin{array}{l}
e_{f f}^{k+1}(\omega)=e_{f f}^{k}(\omega)+\rho(\omega) \cdot \hat{T}_{k}^{-1}(\omega) \cdot e^{k}(\omega) \\
\hat{T}_{k}(\omega)=\hat{T}_{k-1}(\omega) \cdot \Delta \hat{T}_{k-1}(\omega) \\
\Delta \hat{T}_{k-1}(\omega)=\rho^{-1}(\omega)\left(1-\frac{e^{k}(\omega)}{e^{k-1}(\omega)}\right)
\end{array}\right.
$$

If there is no measurement noise $n$ in the system, it can be deduced from (9) that

$$
\Delta T_{k-1}(\omega)=\rho^{-1}(\omega)\left(1-\frac{e^{k}(\omega)}{e^{k-1}(\omega)}\right)
$$

Therefore the estimate value $\left(\Delta \hat{T}_{k-1}(\omega)\right)$ of $\Delta T_{k-1}(\omega)$ is accurate, and it can be deduced that the tracking error of system after $(k+1)$-th iteration is $e^{k+1}(\omega)=0$.

Whereas, in practice, there is the measurement noise $n$ in the system, so there is

$$
\begin{aligned}
\Delta T_{k-1}(\omega)= & \rho^{-1}(\omega)\left(1-\frac{e^{k}(\omega)}{e^{k-1}(\omega)}\right) \\
& -\frac{\rho^{-1}(\omega) S(\omega) \cdot\left(n^{k}(\omega)-n^{k-1}(\omega)\right)}{e^{k-1}(\omega)} \\
\neq & \hat{T}_{k-1}(\omega)
\end{aligned}
$$

It can be seen from (13) that, the estimated value of $\Delta T_{k-1}(\omega)$ is affected by the measurement noise and is not unbiased. In order to make the estimation of $\Delta T_{k-1}(\omega)$ unbiased, the paper proposes a frequency-domain data-driven adaptive ILC (FD-AILC) and its improved iterative learning law is

$$
\left\{\begin{array}{l}
\hat{T}_{k}(\omega)=\hat{T}_{k-1}(\omega) \cdot \Delta \hat{T}_{k-1}(\omega) \\
\Delta \hat{T}_{k-1}(\omega)=\rho^{-1}(\omega)\left[e^{k-1,2}(\omega)-e^{k}(\omega)\right] / e^{k-1}(\omega) \\
e_{f f}^{k}(\omega)=e_{f f}^{k-1}(\omega)+\rho(\omega) \hat{T}_{k-1}^{-1}(\omega) \cdot e^{k-1}(\omega)
\end{array}\right.
$$

where, $e^{k-1}(\omega)$ is the tracking error obtained from the first run of system under the ILC output $e_{f f}^{k-1}(\omega)$, and $e^{k-1,2}(\omega)$ is the tracking error obtained from the second run of system under the ILC output $e_{f f}^{k-1}(\omega)$. In another word, when there is $e_{f f}^{k-1}(\omega)$, the system runs twice during the same iteration, then $e^{k-1}(\omega)$ and $e^{k-1,2}(\omega)$ are obtained respectively. In addition, only $e^{k-1}(\omega)$ is used to calculate the next iteration's ILC output $e_{f f}^{k}(\omega)$.

Furthermore, under the conditions in theorem 1, using the iterative learning control updating formula (14), the FD-AILC method can make the estimation of $T(\omega)$ unbiased. 
Theorem 1: Assuming that $T(\omega)$ and $\hat{T}_{i}(\omega)$ are both stable, $\rho^{-1}(\omega)\left(\left[e^{k-1,2}(\omega)-e^{k}(\omega)\right] / e^{k-1}(\omega)\right)$ is the unbiased estimation of $\Delta T_{k-1}(\omega)$, if

1) $n(\omega)$ and $d_{n}(\omega)$ are both zero mean;

2) The samples of both $n(\omega)$ and $d_{n}(\omega)$ are independent of each other;

3) $n(\omega)$ is independent of $r$ and $d$, and $d_{n}(\omega)$ is independent of $r, n$ and $d_{r}$.

Proof : Firstly, it is defined that

$e_{\tilde{n}}^{k}(\omega)=-S(\omega)\left(n^{k}(\omega)+P(\omega) \cdot d_{n}^{k}(\omega)\right)=-S(\omega) \tilde{n}^{k}(\omega)$

$e^{k}(\omega)$ of second formula in (14) is substituted by (9) and

$$
\begin{aligned}
\Delta \hat{T}_{k-1}= & \frac{\rho^{-1}}{e^{k-1}} \cdot\left[e^{k-1,2}-e^{k-1}+\rho \Delta T_{k-1} e^{k-1}\right. \\
& \left.+S\left(\tilde{n}^{k}-\tilde{n}^{k-1}\right)\right] \\
= & \Delta T_{k-1}+\frac{\rho^{-1}}{e^{k-1}} \cdot\left[e^{k-1,2}-e^{k-1}\right. \\
& \left.+S\left(\tilde{n}^{k}-\tilde{n}^{k-1}\right)\right]
\end{aligned}
$$

According to (6), there is

$$
e^{k-1,2}-e^{k-1}=S\left(-\tilde{n}^{k-1,2}+\tilde{n}^{k-1}\right)
$$

Substituting (17) into (16), there is

$$
\begin{aligned}
\Delta \hat{T}_{k-1}(\omega)= & \Delta T_{k-1}(\omega)+\frac{\rho^{-1}(\omega)}{e^{k-1}(\omega)} \cdot\left(-S(\omega) \tilde{n}^{k-1,2}(\omega)\right. \\
& \left.+S(\omega) \tilde{n}^{k}(\omega)\right) \\
= & \Delta T_{k-1}(\omega)+\frac{\rho^{-1}(\omega)}{e^{k-1}(\omega)} \cdot\left(e_{\tilde{n}}^{k-1,2}(\omega)-e_{\tilde{n}}^{k}(\omega)\right)
\end{aligned}
$$

From condition 2) and 3) of theorem 1, it can be concluded that

$$
\begin{aligned}
E\left[e_{\tilde{n}}^{k-1,2}(\omega) / e^{k-1}(\omega)\right] & =E\left[e_{\tilde{n}}^{k-1,2}(\omega)\right] \cdot E\left[e^{k-1}(\omega)^{-1}\right] \\
E\left[e_{\tilde{n}}^{k}(\omega) / e^{k-1}(\omega)\right] & =E\left[e_{\tilde{n}}^{k}(\omega)\right] \cdot E\left[e^{k-1}(\omega)^{-1}\right]
\end{aligned}
$$

According to the condition 1) of theorem 1, it can be concluded that

$$
\begin{aligned}
E\left[e_{\tilde{n}}^{k-1,2}(\omega)\right] & =E\left[-S(\omega)\left(n^{k-1,2}(\omega)+P(\omega) d_{n}^{k-1,2}(\omega)\right)\right]=0 \\
E\left[e_{\tilde{n}}^{k}(\omega)\right] & =E\left[-S(\omega)\left(n^{k}(\omega)+P(\omega) d_{n}^{k}(\omega)\right)\right]=0
\end{aligned}
$$

Therefore, it can be deduced that

$$
E\left(\Delta \hat{T}_{k-1}(\omega)\right)=\Delta T_{k-1}(\omega)
$$

The theorem 1 is proved. Similar to all other ILC method, according to (9) it is easy to get an expression showing its convergence, as

$$
\left|1-\rho \cdot \Delta T_{k-1}(\omega)\right|<1 \quad i=1,2, \ldots, k
$$

Obviously, this convergence criterion is easy to be met in practice. And the updating of the closed-loop frequencyresponse data cannot be conducted ceaselessly. Therefore, it is necessary to propose a cut-off condition for accelerating convergence. Thus, Theorem 2 is proposed to explain sufficient condition for the algorithm to speed up convergence.
Theorem 2: Under the condition that the updating formula (14) is adopted and theorem 1 is satisfied, and assuming the noise $n$ satisfies the condition of $|\tilde{n}(\omega)| \leq W(\omega)$, then the sufficient condition for the algorithm to ensure that the convergence rate of $k$-th iteration is faster than that of $(k-1)$ th iteration is

$$
\frac{B(\omega)}{A(\omega)}>W(\omega)
$$

where, $A(\omega)$ and $B(\omega)$ are

$$
\begin{aligned}
A(\omega)= & 2 \cdot|S(\omega)| \cdot\left(\left|\rho(\omega) e^{k-1}(\omega)\right|+\left|e^{k-1,2}(\omega)-e^{k}(\omega)\right|\right) \\
B(\omega)= & \left|e^{k}(\omega)\right| \cdot\left|e^{k-1,2}(\omega)-e^{k}(\omega)\right| \\
& -\left|e^{k-1}(\omega)\right| \cdot\left[\left|e^{k-1,2}(\omega)-\rho(\omega) e^{k-1}(\omega)\right|\right. \\
& \left.+\left|(\rho(\omega)-1) e^{k}(\omega)\right|\right]
\end{aligned}
$$

Proof: The accelerated convergence can be depicted as

$$
\frac{\left|e^{k+1}(\omega)\right|}{\left|e^{k}(\omega)\right|}<\frac{\left|e^{k}(\omega)\right|}{\left|e^{k-1}(\omega)\right|}
$$

According to equation (9), above expression can be rewritten into

$$
\frac{\left|1-\rho(\omega) \Delta T_{k}(\omega)\right|}{\left|1-\rho(\omega) \Delta T_{k-1}(\omega)\right|}<1
$$

According to (9), there is

$$
1-\rho(\omega) \cdot \Delta T_{k-1}=\frac{e^{k}(\omega)-\left(e_{\tilde{n}}^{k}(\omega)-e_{\tilde{n}}^{k-1}(\omega)\right)}{e^{k-1}(\omega)}
$$

Combining the definition of $\Delta T_{k}(\omega)$ in (4) and the first formula in the updating law (14), the detailed derivation is omitted due to the space restrictions so the derived result is as follows.

$$
\Delta T_{k}(\omega)=\frac{\Delta T_{k-1}(\omega)}{\Delta \hat{T}_{k-1}(\omega)}
$$

$\Delta T_{k-1}(\omega)$ can be obtained through (27). Substituting $\Delta T_{k-1}(\omega), \Delta \hat{T}_{k-1}(\omega)$ of (14), (27) and (28) into (26), it can be obtained that

$$
\begin{array}{r}
\frac{\left|e^{k-1,2}-\rho e^{k-1}+(\rho-1) e^{k}+\rho\left(e_{\tilde{n}}^{k}-e_{\tilde{n}}^{k-1}\right)\right|}{\left|e^{k-1,2}-e^{k}\right|} \\
<\frac{\left|e^{k}-\left(e_{\tilde{n}}^{k}-e_{\tilde{n}}^{k-1}\right)\right|}{\left|e^{k-1}\right|}
\end{array}
$$

For the left and right sides of above expression, there is

$$
\begin{aligned}
f_{\text {left }} & \leq \frac{\left|e^{k-1,2}-\rho e^{k-1}+(\rho-1) e^{k}\right|+2 \rho W \cdot|S|}{\left|e^{k-1,2}-e^{k}\right|}=M \\
f_{\text {right }} & \geq \frac{\left|e^{k}\right|-2 W \cdot|S|}{\left|e^{k-1}\right|}=N
\end{aligned}
$$

It is noted that in (29) and (30), ( $\omega)$ of all the variables are omitted due to the space restrictions. From (30), it can be seen that $M<N$ is the sufficient condition for (29) to be true. As a result, (23) and (24) can be deduced from $M<$ $N$. The unbiased estimation and conditions for accelerating convergence of the FD-AILC method have been discussed in 
the above contents. It is noted that Theorem 2 can be used for deciding whether update $\hat{T}_{k}(\omega)$, that is stopping updating of $\hat{T}_{k}(\omega)$ when the sufficient condition is not satisfied.

Remark 1: If the external disturbance only consists of repetitive disturbance, $W(\omega)$ in (23) will be the supremum of $|n(\omega)|$.

The detailed algorithm flow of FD-AILC is given below.

Step 1: The system is run independently twice with the same ILC output $e_{f f}^{i}(t)$, and the two data of tracking error $e^{i, 1}(t)$ and $e^{i, 2}(t)$ are obtained repectively;

Step 2: The FFT calculations are done for the data of $e^{i, 1}(t)$ and $e^{i, 2}(t)$, and their corresponding frequency spectrum $e^{i, 1}(\omega)$ and $e^{i, 2}(\omega)$ are obtained;

Step 3: Decide whether update $\hat{T}_{i}(\omega)$ or not according to the theorem 2, if yes enter Step 4 , otherwise $\hat{T}_{i}(\omega)=\hat{T}_{i-1}(\omega)$ is set and enter Step 5 ;

Step 4: According to the updating method (14), the closedloop system frequency response is updated to obtain $\hat{T}_{i}(\omega)$;

Step 5: The ILC output $e_{f f}^{i+1}(\omega)$ is updated by using the equation $e_{f f}^{i+1}(\omega)=e_{f f}^{i}(\omega)+\hat{T}_{i}^{-1}(\omega) \cdot e^{i}(\omega)$;

Step 6: The IFFT calculations are done for the data of $e_{f f}^{i+1}(\omega)$ to obtain $e_{f f}^{i+1}(t)$;

Step 7: $i=i+1$ is set and return to Step 1 .

\section{Results}

To illustrate the proposed FD-AILC method and evaluate its validity, numerical simulation and experimental test on a wafer stage are implemented in this section.

\section{A. Simulation Results}

\section{1) Simulation Setup}

The plant model in simulation is designed as follow, which is one of the most common dynamical models of the mechanical systems,

$$
P(s)=\frac{1}{m s^{2}}+\frac{K}{s^{2}+2 \xi \omega_{n}+\omega_{n}^{2}}
$$

where $P(s)$ is the transfer function of the controlled object. To more visibly observe the effectiveness and superiority of the proposed method, the controlled object is designed with lower flexible modal frequency and smaller mass which can depict the low-weight flexible property of the next-generation precision stage. So the parameters of the plant model in simulation are set as $m=5.0 \mathrm{Kg}, K=0.09, \xi=0.01$ and $\omega_{n}=2 \pi \cdot 500.5$ respectively. The sampling period of the system is $\mathrm{T}_{\mathrm{s}}=200 \mu \mathrm{s}$. In addition, the time delay of the system is set as $\tau=200 \mu \mathrm{s}$, which equals $1 \mathrm{~T}_{\mathrm{s}}$.

The control system is designed according to Fig. 1, where the feedback controller is a PI controller with a lead correction shown in (32). The system closed-loop bandwidth is defined as the frequency point at $-3 d B$ of the complementary sensitivity function. So the bandwidth of the control system is $91 \mathrm{~Hz}$.

$$
C_{f b}(s)=1.3866 \times 10^{7} \times \frac{0.0159 s+1}{s} \times \frac{0.0071 s+1}{0.0014 s+1}
$$

where $s$ denotes the Laplace operator.
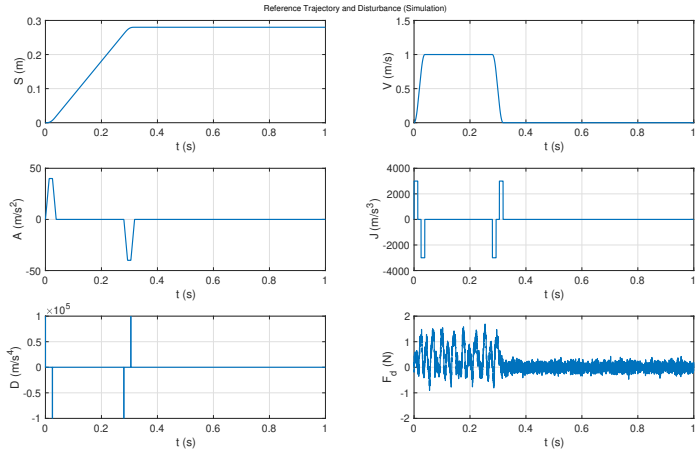

Fig. 2: The reference trajectory and external disturbance in the simulation.

Fig. 2 shows the curves of the reference trajectory and external disturbance used in simulation. The reference trajectory in simulation is selected as a fourth-order multisegment polynomial trajectory, with constraints on first to fourth derivatives. The parameters are the displacement $\mathrm{s}=0.28 \mathrm{~m}$, the maximum velocity $\mathrm{v}=1 \mathrm{~m} / \mathrm{s}$, the maximum acceleration $\mathrm{a}=40 \mathrm{~m} / \mathrm{s}^{2}$, the maximum jerk $\mathrm{J}=3000 \mathrm{~m} / \mathrm{s}^{3}$, and the maximum snap $\mathrm{D}=5 \times 10^{7} \mathrm{~m} / \mathrm{s}^{4}$ respectively. Because in most applications that this paper concerns, the system external disturbance exhibit repetitive property during the iterative process, the disturbance used in simulation is designed as a repetitive disturbance force. The data of the disturbance consists of the cogging force and the cable force, which is collected on a practical stage driven by the linear motor.

The measurement noise is set as Gaussian white noise whose amplitude is $1 \mathrm{~nm}$. Additionally, it is necessary to design an initial frequency-response data $\hat{T}_{0}(\omega)$ used for the first iterative trial. In the simulation test, the real value of $T(\omega)$ is known. So $\hat{T}_{0}(\omega)$ is designed as $\hat{T}_{0}(\omega)=c_{\text {rand }}(\omega) \cdot T(\omega)$, where $c_{\text {rand }}(\omega)$ is a random number distributed uniformly in $(0.75,1.25)$. Apart from above setup, the positive real regulator $\rho(\omega)$ in the updating formula (14) is selected as 0.7 .

To further illustrate its superiority compared with other ILC method, the FD-ILC mentioned in Section II and the ILC method based on the transfer function (TF-ILC) proposed in [11] are also tested in simulation. To provide a fair comparison, the same $\hat{T}_{0}(\omega)$ is used for $\hat{T}(\omega)$ of (2) for FD-ILC. As for TF-ILC method, the learning law $C_{L_{-} T F}$ is shown as follow,

$$
C_{L_{-} T F}(s)=K_{T F} \cdot Q_{T F}(s) \cdot T^{-1}(s)
$$

where $s$ denotes the Laplace operator, $K_{T F}$ is the learning gain set as $0.7, Q_{T F}(s)$ is a second order low-pass filter with cut-off frequency of $2000 \mathrm{~Hz}$ and damping ratio of 1 , and $T(s)$ is set as the transfer function of the real closed-loop system.

\section{2) Simulation Results}

Firstly, an evaluating criterion Error- $\mathrm{J}$ is defined to present the 2-norm of the tracking error, that is

$$
J_{k}=\left\|e_{k}\right\|_{2}
$$

Under the same simulating conditions, FD-ILC, TF-ILC and the proposed FD-AILC are performed for 10 iterations 

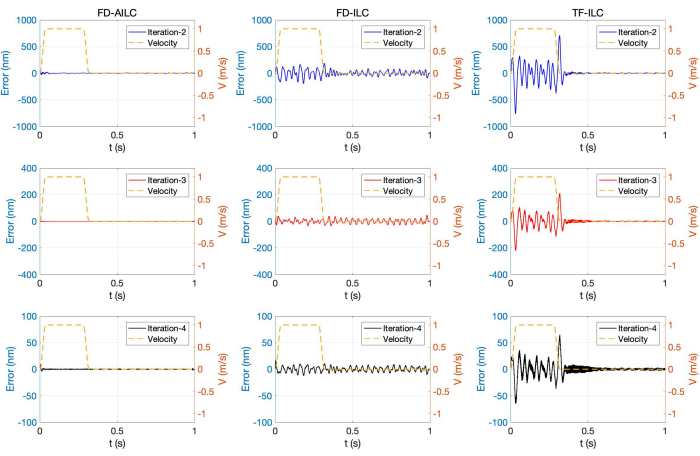

Fig. 3: The tracking errors comparison among the FD-AILC, FD-ILC and TF-ILC. In each subfigure, the left scale shows the range of the error and the right scale shows the range of the velocity.

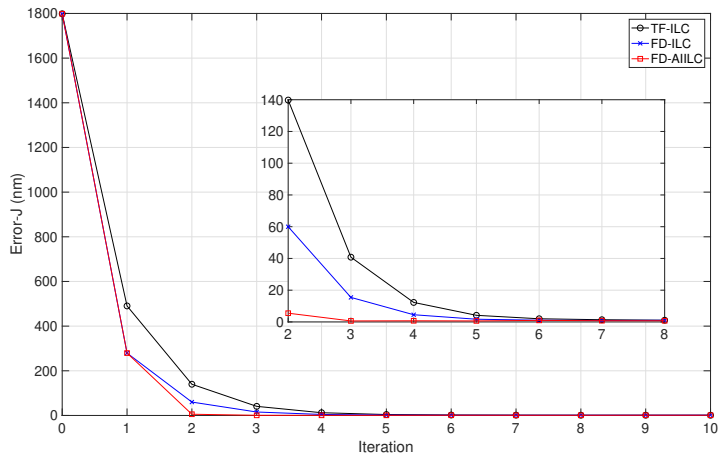

Fig. 4: The Error-J comparison between FD-AILC, FD-ILC and TF-ILC.

respectively. Fig. 3 shows the comparative result of the timedomain tracking error and Fig. 4 shows the comparative result of Error-J.

It can be observed from Fig. 3 that after performing the same iterations, the tracking errors of the proposed method are smaller than those of the FD-ILC and TF-ILC, which proofs the effectiveness of FD-AILC and verifies that the proposed method is better than another two methods in improving the system tracking performance. Fig. 4 shows that after 3iterations through the FD-AILC the system tracking error can achieve convergence to the minimum value, while both FDILC and TF-ILC need about 6-iterations to achieve convergence. Therefore, result in Fig. 4 further demonstrates that introducing an adaptive algorithm for updating the iterative learning law facilitates improving the convergence speed.

In the simulation test, the estimating result of the closedloop frequency response can be observed, because the real value of $T(\omega)$ is known. To further evaluating the proposed method, the estimation result of $T(\omega)$ is shown in Fig. 5, and the updated $\Delta T(\omega)$ is shown in Fig. 6 and Fig. 7. $\Delta T(\omega)$ is defined in (4).

From Fig. 5, it can be observed that the curve of $\hat{T}(\omega)$ becomes smooth as the increasing of the iteration, which illustrates that FD-AILC method can effectively update $\hat{T}(\omega)$
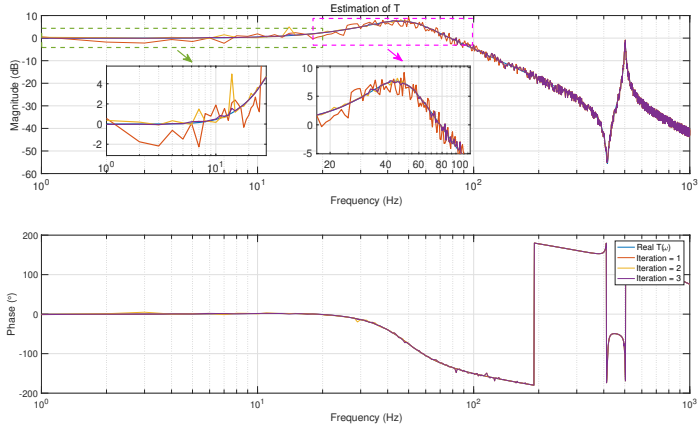

Fig. 5: The simulation result of $\hat{T}(\omega)$ for FD-AILC method.

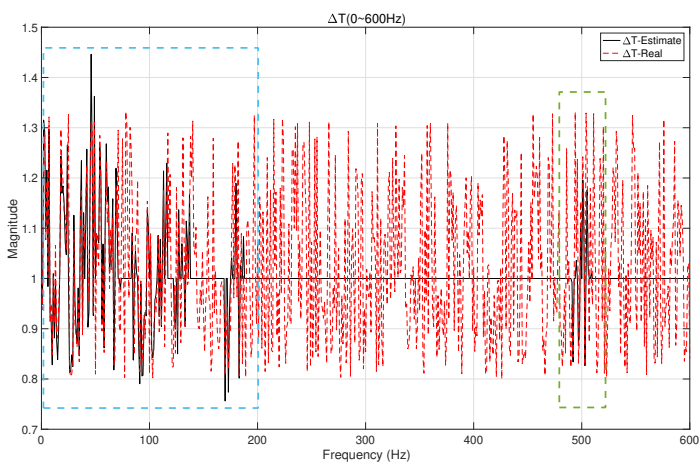

Fig. 6: $\Delta T$ in frequency domain between $0 \sim 600 H z$.

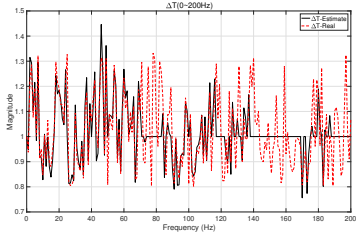

(a) $0 \sim 200 H z$

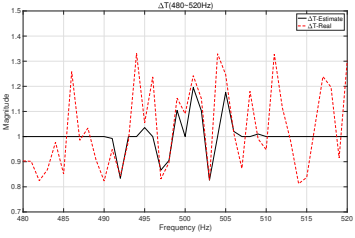

(b) $480 \sim 520 \mathrm{~Hz}$
Fig. 7: Different scale figures of $\Delta T$ in $0 \sim 200 \mathrm{~Hz}$ (a) and $480 \sim 520 H z$ (b).

and then help improve convergence speed. Seen from Fig. 6 and Fig. 7, instead of updating the data of the whole frequency band, the algorithm updates parts of $\Delta T(\omega)$. The updated data are mainly distributed in the frequency band of $0 \sim 200 \mathrm{~Hz}$ and $480 \sim 520 \mathrm{~Hz}$. Above result needs to be analyzed combining with the feedback system closed-loop frequency response showing in Fig. 8.

From Fig. 7 and Fig. 8, it can be concluded that using the proposed method can update the data of $\Delta T(\omega)$ corresponding to the frequency bands in the red rectangular of Fig. 8. The amplitudes of the system tracking errors corresponding to the frequency bands outside the red rectangular are too small which is close to the measurement noise. As a result, according to the updating cut-off condition in (23) the proposed datadriven adaptive algorithm does not update the frequency response at the frequency that its corresponding tracking error is small enough. Therefore, the above analysis further 

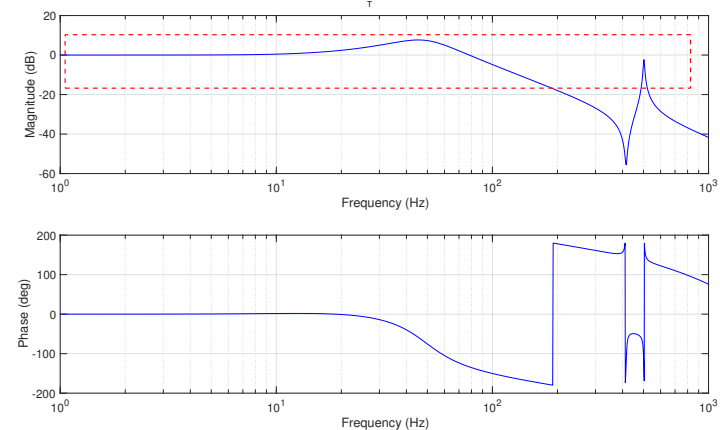

Fig. 8: $T$ in frequency domain between $0 \sim 1000 H z$.

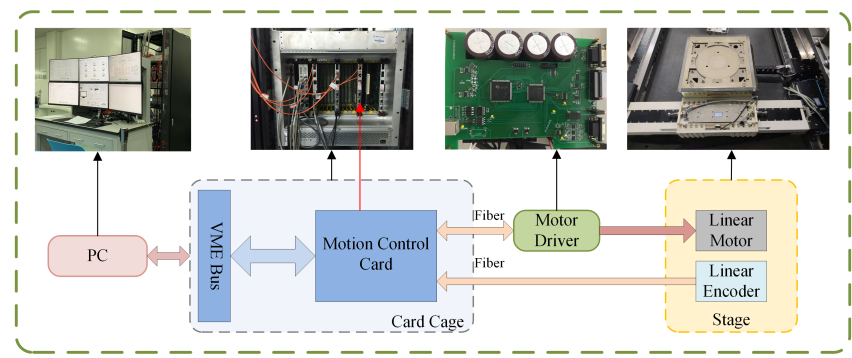

Fig. 9: Block diagram of the experimental setup.

illustrates the effectiveness of the proposed adaptive algorithm in improving the frequency-response data used in feedforward compensation.

\section{B. Experimental Results}

\section{1) Experimental Setup}

To better illustrate the effectiveness of the proposed method, experiments were performed on a linear motor of the wafer stage. It is noted that the controlled object of the experiment is different from the simulation, because the practical experimental platform is without the property of low-weight and lower flexible modal frequency. But the comparative experimental results can still illustrate the strength of the proposed method. The experimental setup is shown in Fig. 9. The real-time operating system is selected as VxWorks. The mainboard and the motion control (MC) card are integrated into a VME64x card cage from the Germany company ELMA. The flow of the control signal and data signal between the PC and the control card cage are realized through the network cable and serial port respectively. The MC card sends the control command to the motor driver via the fiber. Similarly, the data of the linear encoder is transmitted through the fiber. The motor driver can make the bandwidth of the current-loop achieve $2000 \mathrm{~Hz}$ and its peak current is $60 \mathrm{~A}$. The wafer stage is mounted on an air bearing with $400 k P a$ air pressure. The position sensor is a Heidenhain linear incremental encoder with the effective resolution of $0.05 \mu \mathrm{m}$ and the maximum velocity of $0.3 \mathrm{~m} / \mathrm{s}$. And the control methods are implemented by $\mathrm{C}$ language on a digital signal processor (DSP). The sampling period is $\mathrm{T}_{\mathrm{s}}=200 \mu \mathrm{s}$. The feedback controller $C_{f b}$ is a PI controller with a lead correction that is similar to (32).
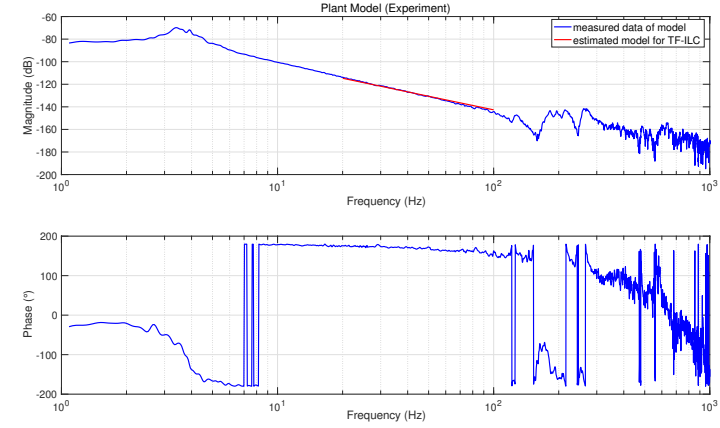

Fig. 10: The measured frequency response of the plant model in experiment.

The closed-loop system is excited by a preset time series, which structure is the same as the reference trajectory in the simulation test as shown in Fig. 2. Its parameters are $\mathrm{s}=0.1 \mathrm{~m}, \mathrm{v}=0.25 \mathrm{~m} / \mathrm{s}, \mathrm{a}=10 \mathrm{~m} / \mathrm{s}^{2}, \mathrm{~J}=800 \mathrm{~m} / \mathrm{s}^{3}$, $\mathrm{D}=1 \times 10^{5} \mathrm{~m} / \mathrm{s}^{4}$ and $\mathrm{H}=1 \times 10^{8} \mathrm{~m} / \mathrm{s}^{5}$ respectively. Notably, to keep the same starting point of the linear motor in every iteration, the motor does the reciprocating motion, where the reciprocating trajectory is the same.

Similar to the simulation test, the proposed FD-AILC, FD-ILC and TF-ILC are compared. For TF-ILC method, an approximate model of the plant is required so a dual integral model is adopted to fit the measured plant model shown in Fig. 10. The fitted result is expressed as $G_{e s t}=\frac{1}{34.3775 s^{2}}$. The learning law of TF-ILC in the experiment is the same as that in simulation and its parameters are set as: 1)the learning gain $K_{T F}=0.7 ; 2$ )the second-order low-pass filter with a cutoff frequency of $100 \mathrm{~Hz}$ and with a damping ratio of 1 . Also, it is worth noting that the initial frequency response $\hat{T}_{0}(\omega)$ is required both in FD-AILC and FD-ILC. In FD-ILC, it will be used for all iterations, whereas for FD-AILC, it will only be used for the first iteration and the data will be updated in other iterations. The inversion of the initial frequency response $\hat{T}_{0}^{-1}(\omega)$ is obtained through a frequency-response test on the practical stage and its result is shown in the Bode diagram in Fig. 11, where the blue line is the practically measured data of $\hat{T}_{0}^{-1}(\omega)$. Because the measured high-frequency data are greatly affected by the measurement noise, only the frequency range data showed as the red line in Fig. 11 are used in the iterative methods.

\section{2) Experimental Results}

The FD-AILC is performed for 5 iterations on the experimental stage and the result is shown in Fig. 12.

From Fig. 12, a conclusion can be drawn that the tracking error of the system drops significantly with the increasing of the iteration times, which confirms that the proposed method is effective in improving the tracking performance. Since there is no feedforward compensation when running the system for the first time, the tracking error is relatively large which is over $50 \mu \mathrm{m}$. After the first iteration, the tracking error reduced to about $10 \mu \mathrm{m}$. However, the effectiveness of updating the learning law is unable to be verified through the result in Fig. 12. Therefore, comparative experiments are required to 

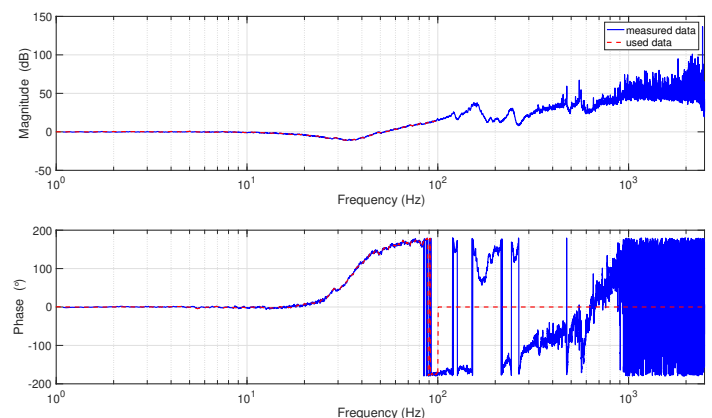

Fig. 11: Initial frequency-response data of closed-loop control system inversion $\hat{T}_{0}^{-1}(\omega)$ in experiment.

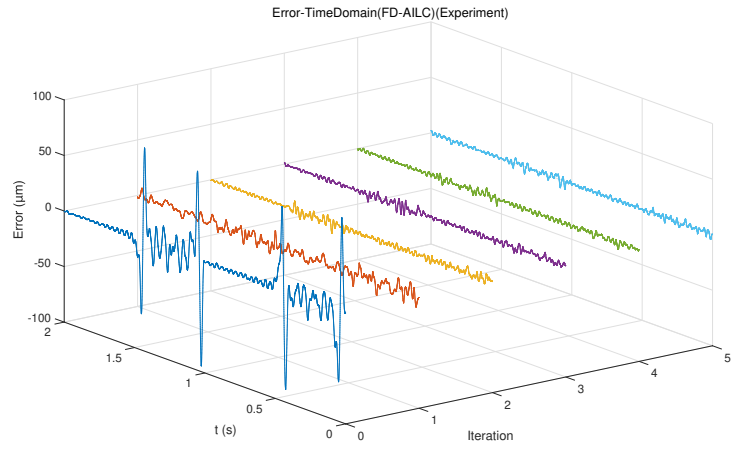

Fig. 12: The experimental result of FD-AILC method.

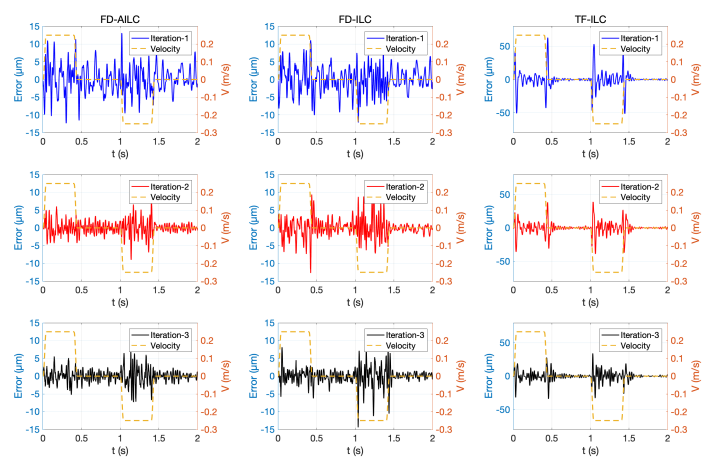

Fig. 13: The tracking errors comparison among the FD-AILC, FD-ILC and TF-ILC. In each subfigure, the left scale shows the range of the error and the right scale shows the range of the velocity.

further prove the effectiveness and superiority of the proposed method. Then the FD-ILC method and TF-ILC method are run for 5 iterations as well under the same experimental conditions and results are shown in Fig. 13, Fig.14 and Fig.15. It is noted that Error-J is defined in (34).

In Fig. 12, Fig. 13 and Fig. 14, Iteration $=0$ presents the system is run without any iterative learning output. From the first iteration, the FD-ILC and TF-ILC use the frequencyresponse data $\hat{T}_{0}^{-1}(\omega)$ shown in Fig. 11 and transfer function model fitted by the dual integral model respectively to

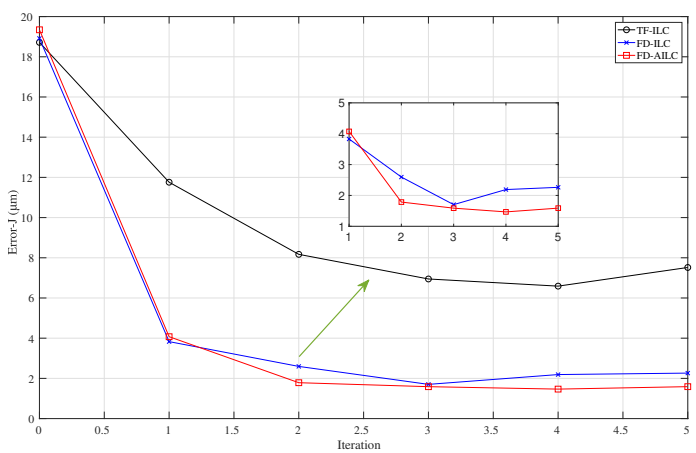

Fig. 14: The Error-J comparison among the FD-AILC method, FD-ILC method and TF-ILC.
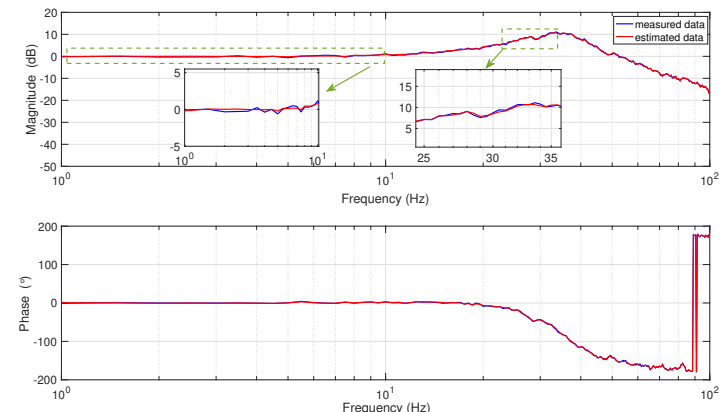

Fig. 15: The experimental result of $\hat{T}(\omega)$ for FD-AILC method.

calculate the ILC output. Whereas, the proposed FD-AILC will use $\hat{T}_{0}^{-1}(\omega)$ only for the first iterative trial and update it through the adaptive learning law shown in (14) according to conditions in (23) for other trials. From these experimental results, the following conclusions and analysis can be drawn.

1) After the first iteration, the maximum tracking error of both the FD-AILC and FD-ILC are very close and below $15 \mu \mathrm{m}$, which is on account of the same frequency-response data used in the first iteration. Whereas the maximum tracking error of the TF-ILC method is about $50 \mu \mathrm{m}$, which is over three times bigger than those of the other two methods. This observation is due to that the approximation model of the controlled object used in TF-ILC is unable to describe the real characteristics of the system accurately, which proves that using the frequency-response information can effectively avoid the above problem.

2) From Fig. 14, it can be confirmed that TF-ILC is helpful to improve the tracking accuracy after several iterations. However, the evaluation indicator Error-J does not decrease for all the time and it seems to tend to enlarge. The problem of the model mismatch for the TF-ILC method is mainly responsible for the above phenomenon. The model mismatch would probably lead to that the system does not satisfy the convergence condition of the iterative learning algorithm at some frequency points, which further results in that the system tracking error would have an upward trend.

3) From Fig. 13, it can be concluded that both after the 
second iteration and third iteration, the tracking error of the FD-AILC method is smaller than that of the FD-ILC method, which can demonstrate that updating the iterative learning law benefits to improve the tracking performance.

4) From Fig. 13 and Fig. 14, it can be observed that after 3 iterations, using the proposed method the maximum tracking error of the system can converge to about $7 \mu \mathrm{m}$, whereas using the FD-ILC the maximum tracking error only converges to about $15 \mu \mathrm{m}$. Fig. 14 further illustrates that the proposed FDAILC is better in improving the convergence speed and verifies the effectiveness of the proposed adaptive iterative law.

5) From Fig. 14, it can be noted that the Error-J of the FDILC is slightly larger than that of the FD-AILC and it has an upward trend after 3 iterations, whereas in simulation the curve of Error-J for FD-ILC does not show this trend. Analyzing this founding, there are two possible reasons responsible for it. Firstly, similar to conclusion 2) it is caused by the mismatch of the frequency-response data. For FD-ILC, using an inaccurate frequency-response data could cause incorrect feedforward compensation value of some frequency points. This mismatch of the frequency-response data will result in slight deterioration of the compensation. Another reason is that in the experimental tests, there are frequency-response data which are not satisfying the condition of convergence at some frequency points, which would also lead to slight deterioration of the compensation. As to the FD-AILC, it can avoid the above possible problems through the adaptive iterative law, which further verifies the ability of the proposed method for keeping the system stability.

6) Fig. 15 shows the experimental result of $\hat{T}(\omega)$ using FD-AILC method. The curve of the estimated result looks smoother than the curve of the measured data, especially in the low-frequency range. This observation can verify the validity of FD-AILC on self-adaptive updating the frequency-response model to some extent. However, the improvement of $\hat{T}(\omega)$ is not so significant due to that the practical experimental platform is not with the complicated low-frequency characteristics.

Consequently, the proposed FD-AILC can effectively improve both the tracking accuracy and the convergence speed, as well as avoiding performance deterioration caused by the model mismatch. In addition, since there is no plant model required, the proposed method is helpful to reduce the workload of designing the control system.

\section{CONCLUSION}

This paper addresses practical problems of the future lightweight flexible stages, including the high requirement of the motion performance, the fast convergence rate and the complicated low-frequency characteristics. First, a modelfree frequency-domain data-driven adaptive ILC method has been established, which enables the possibility of improving the tracking performance by updating iterative learning law during the iterative process adaptively. Theoretical analysis indicates the updating algorithm could obtain an unbiased estimation of the frequency response. Subsequently, the criteria for accelerating convergence are derived. The numerical simulation and experimental results with comparison fully illustrate the benefits of the proposed model-free ILC approach, listed as (1)its ability to achieve higher tracking accuracy; (2)its superiority to increasing convergence speed; (3)its advantage of reducing the workload of designing the control system. Finally, the future work can be predicted towards solving problems of tracking control with non-repeated trajectory, which is more general in industrial applications.

\section{REFERENCES}

[1] K. Saiki, A. Hara, K. Sakata, and H. Fujimoto, "A study on high-speed and high-precision tracking control of large-scale stage using perfect tracking control method based on multirate feedforward control," IEEE Transactions on Industrial Electronics, vol. 57, no. 4, pp. 1393-1400, 2009.

[2] P. Niranjan, S. Karinka, and K. Sairam, "Development of precision positioning stage," Journal of Advanced Manufacturing Systems, vol. 18, no. 01, pp. 103-111, 2019.

[3] J.-C. Shen, Q.-Z. Lu, C.-H. Wu, and W.-Y. Jywe, "Sliding-mode tracking control with DNLRX model-based friction compensation for the precision stage," IEEE/ASME Transactions on Mechatronics, vol. 19, no. 2, pp. 788-797, 2013.

[4] H. Butler, "Magnetic disturbance compensation for a reticle stage in a lithographic tool," Mechatronics, vol. 23, no. 6, pp. 559-565, 2013.

[5] L. Liu, S. Tian, D. Xue, T. Zhang, and Y. Chen, "Industrial feedforward control technology: a review," Journal of Intelligent Manufacturing, vol. 30, no. 8, pp. 2819-2833, 2019.

[6] F. Song, Y. Liu, J.-X. Xu, X. Yang, and Q. Zhu, "Data-driven iterative feedforward tuning for a wafer stage: A high-order approach based on instrumental variables," IEEE Transactions on Industrial Electronics, vol. 66, no. 4, pp. 3106-3116, 2018.

[7] H. Butler, "Adaptive feedforward for a wafer stage in a lithographic tool,' IEEE Transactions on Control Systems Technology, vol. 21, no. 3, pp. 875-881, 2012.

[8] L. Li, Y. Liu, L. Li, and J. Tan, "Kalman-filtering based iterative feedforward tuning in presence of stochastic noise: with application to a wafer stage," IEEE Transactions on Industrial Informatics, 2019.

[9] D. Bruijnen and N. van Dijk, "Combined input shaping and feedforward control for flexible motion systems," in 2012 American Control Conference (ACC), pp. 2473-2478. IEEE, 2012.

[10] B. Chu, D. H. Owens, and C. T. Freeman, "Iterative learning control with predictive trial information: convergence, robustness, and experimental verification," IEEE Transactions on Control Systems Technology, vol. 24, no. 3, pp. 1101-1108, 2015.

[11] H. Hashimoto, P.-H. Yang, K. Hirano, B. YUAN, X.-F. YANG, Y. ITAKURA, A. YAMAGUCHI, R. KAWAGUCHI, and S. MAKINOUCHI, "Precision and settling time improvement for the wafer stage of lithography scanners with iterative learning control," in Proceedings of the 1st International Conference on Positioning Technology, pp. 9196, 2004.

[12] X. Fu, X. Yang, and Z. Chen, "A new linear motor force ripple compensation method based on inverse model iterative learning and robust disturbance observer," Complexity, vol. 2018, 2018.

[13] T. Shiraishi and H. Fujimoto, "Positioning control for piezo scanner using multirate perfect inverse model based iterative learning control," in 2010 IEEE/ASME International Conference on Advanced Intelligent Mechatronics, pp. 993-998. IEEE, 2010.

[14] B. G. Dijkstra and O. H. Bosgra, "Convergence design considerations of low order q-ilc for closed loop systems, implemented on a high precision wafer stage," in Proceedings of the 41st IEEE Conference on Decision and Control, 2002., vol. 3, pp. 2494-2499. IEEE, 2002.

[15] S. Mishra and M. Tomizuka, "Projection-based iterative learning control for wafer scanner systems," IEEE/ASME Transactions on Mechatronics, vol. 14, no. 3, pp. 388-393, 2009.

[16] T. Oomen, R. van Herpen, S. Quist, M. van de Wal, O. Bosgra, and M. Steinbuch, "Connecting system identification and robust control for next-generation motion control of a wafer stage," IEEE Transactions on Control Systems Technology, vol. 22, no. 1, pp. 102-118, 2013.

[17] B. Xu-Hui, Y. Fa-Shan, H. Zhong-Sheng, and W. Fu-Zhong, "Iterative learning control for a class of linear discrete-time switched systems," Acta Automatica Sinica, vol. 39, no. 9, pp. 1564-1569, 2013.

[18] X. Yang, "A PD-type iterative learning control for a class of switched discrete-time systems with model uncertainties and external noises," Discrete Dynamics in Nature and Society, vol. 2015, 2015. 
[19] H.-S. Ahn, Y. Chen, and K. L. Moore, "Iterative learning control: Brief survey and categorization," IEEE Transactions on Systems, Man, and Cybernetics, Part C (Applications and Reviews), vol. 37, no. 6, pp. 1099$1121,2007$.

[20] S. Tien, Q. Zou, and S. Devasia, "Iterative control of dynamics-couplingcaused errors in piezoscanners during high-speed afm operation," IEEE Transactions on Control Systems Technology, vol. 13, no. 6, pp. 921931, 2005

[21] K.-S. Kim and Q. Zou, "A modeling-free inversion-based iterative feedforward control for precision output tracking of linear time-invariant systems," IEEE/ASME Transactions on Mechatronics, vol. 18, no. 6, pp. $1767-1777,2012$.

[22] T. D. Son, G. Pipeleers, and J. Swevers, "Multi-objective iterative learning control using convex optimization," European Journal of Control, vol. 33, pp. 35-42, 2017.

[23] W. Paszke, K. Patan, and E. Rogers, "Experimentally verified multiobjective iterative learning control design with frequency domain specifications," in 2018 European Control Conference (ECC), pp. 3143-3148. IEEE, 2018.

[24] J. Zhu, Z. Cai, J. Zhao, and Y. Wang, "Improved frequency domain iterative learning control applied to trajectory tracking," in 2017 11th Asian Control Conference (ASCC), pp. 210-215. IEEE, 2017.

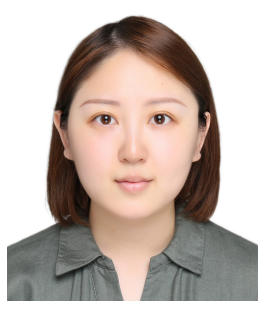

Xuewei Fu received the B.S. degree in automation and M.S. degree in control science and engineering from the Harbin Institute of Technology, Harbin, China, in 2015 and 2017, respectively. She is currently working toward the Ph.D. degree in microelectronics and solid-state electronics with the Fudan University, Shanghai, China.

Her research interests include iterative learning control and disturbance suppression with applications in high-precision mechanical servo systems.

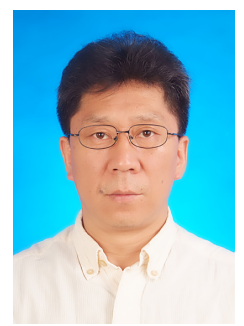

Xiaofeng Yang received his M.Sc. degree in Automatic Control from Northeastern University, Shenyang, China, in 1989 and the Ph.D. degree in Mechanical Engineering from Sophia University, Tokyo, Japan, in 1997.

From 1997 to 2010, he was with the Precision Equipment Company of Nikon Corporation, Kumagaya, Japan, where he worked in the field of nano-precision wafer and reticle stage development for semiconductor lithography machines.

$\mathrm{He}$ is currently a full professor in the School of Microelectronics at Fudan University, Shanghai, China. His research interests include design and implementation of control systems for ultra precision positioning devices, design and application of advanced motors, and vibration isolation systems for precision machines.

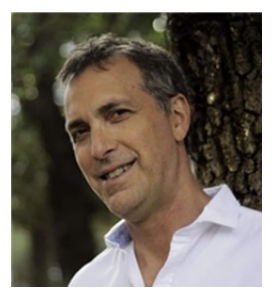

Pericle Zanchetta (F'19) received the M.Eng. degree in electronic engineering and the Ph.D. degree in electrical engineering from the Technical University of Bari, Bari, Italy, in 1993 and 1997 , respectively.

In 1998, he became an Assistant Professor of Power Electronics at the Technical University of Bari. In 2001, he became a Lecturer in Control of Power Electronics Systems with the Power Electronics, Machine and Control (PEMC) Research Group, University of Nottingham, Nottingham, U.K., where he is currently a Professor in Control of Power Electronics Systems.

He has published more than 330 peer-reviewed papers. His research interests include control and optimization of power converters and drives, matrix, and multilevel converters. Dr. Zanchetta has been the Chair of the IEEE-IAS Industrial Power Converter Committee IPCC and he is currently the Transactions Review Chair for IPCC. He is also the Vice-Chair of the IEEE-IAS Industrial Power Conversion Systems Department.

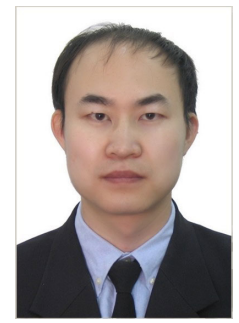

Yang Liu (M'12) received the B.S. degree in automation and the M.S. and Ph.D. degrees in control science and engineering from the Harbin Institute of Technology, Harbin, China, in 2004 2006 , and 2011, respectively.

From 2014 to 2015, he was a Research Fellow with Queen's University Belfast. He is currently an Associate Professor with Harbin Institute of Technology. His current research interests include system identification and precision motion control.

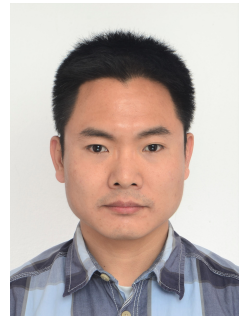

Chenyang Ding was born in 1981 . He received the B.S. degree (Electrical Engineering) from Xian Jiaotong University in 2003 and the M.S. degree (Mechatronics) from National University of Singapore in 2005. In 2013, he received the Ph.D. degree from Control Systems Group, Electrical Engineering, Eindhoven University of Technology based on the thesis Vibration isolation control of a contactless electromagnetic suspension system.

He worked on dynamics and control of precision mechatronic systems in high-tech industry in Eindhoven, the Netherlands for a few years, and in 2019, he was appointed Associate Professor in Academy for Engineering \& Technology, Fudan University, Shanghai, China.

His main interest is dynamic modeling and precision control of piezo actuators, maglev systems, and vibration isolation systems.

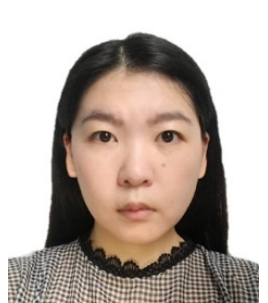

Mi Tang (M'16) received the M.Sc. degree in electrical engineering and Ph.D. degree in electrical and electronic engineering from the University of Nottingham, Nottingham, U.K., in 2012 and 2017, respectively.

She is currently a Research Fellow with the Power Electronics, Machine and Control Group, University of Nottingham, Nottingham, U.K.

Her research interests include deadbeat control, fault-tolerant control and repetitive control.

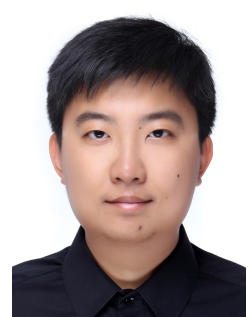

Zhenyu Chen received the B.S. degree in automation and M.S. degree in control science and engineering from the Harbin Institute of Technology, Harbin, China, in 2014 and 2016, respectively. $\mathrm{He}$ is currently working toward the Ph.D. degree in instrument science and technology with the Harbin Institute of Technology, Harbin, China.

His research interests include precision motion control, iterative learning control, and active vibration control with applications in highprecision mechanical servo systems. 